\title{
Integrating the HERMES spectrograph for the AAT
}

\author{
Jeroen Heijmans ${ }^{\mathrm{a}}$, Martin Asplund ${ }^{\mathrm{b}}$, Sam Barden ${ }^{\mathrm{a}, \mathrm{c}}$, Michael Birchall ${ }^{\mathrm{a}}$, Daniela Carollo ${ }^{\mathrm{g}}$, Joss Bland \\ Hawthorn $^{\mathrm{d}}$, Jurek Brzeski ${ }^{\mathrm{a}}$, Scott Case ${ }^{\mathrm{a}}$, Vladimir Churilov ${ }^{\mathrm{a}}$, Matthew Colless ${ }^{\mathrm{a}}$, Robert Dean ${ }^{\mathrm{a}}$, \\ Gayandhi De Silva $^{\mathrm{a}}$, Tony J. Farrell ${ }^{\mathrm{a}}$, Kristin Fiegert ${ }^{\mathrm{a}}$, Ken Freeman ${ }^{\mathrm{b}}$, Luke Gers ${ }^{\mathrm{a}}$, Michael \\ Goodwin $^{\mathrm{a}}$, Doug Gray ${ }^{\mathrm{a}}$, Ron Heald ${ }^{\mathrm{a}}$, Anthony Heng ${ }^{\mathrm{a}}$, Damien Jones ${ }^{\mathrm{e}}$, Chiaki Kobayashi ${ }^{\mathrm{f}}$, Urs \\ Klauser $^{\mathrm{a}}$, Yuriy Kondrat ${ }^{\mathrm{a}}$, Jon Lawrence ${ }^{\mathrm{a}}$, Steve Lee ${ }^{\mathrm{a}}$, Darren Mathews ${ }^{\mathrm{a}}$, Don Mayfield ${ }^{\mathrm{a}}$, Stan \\ Miziarski ${ }^{a}$, Guy Monnet ${ }^{a}$, Rolf Muller ${ }^{\mathrm{a}}$, Naveen Pai ${ }^{\mathrm{a}}$, Robert Patterson ${ }^{\mathrm{a}}$, Ed Penny ${ }^{\mathrm{a}}$, David Orr ${ }^{\mathrm{a}}$, \\ Andrew Sheinis ${ }^{\mathrm{a}^{*}}$, Keith Shortridge ${ }^{\mathrm{a}}$, Scott Smedley ${ }^{\mathrm{a}}$, Greg Smith ${ }^{\mathrm{a}}$, Darren Stafford ${ }^{\mathrm{a}}$, Nicholas \\ Staszak $^{\mathrm{a}}$, Minh Vuong ${ }^{\mathrm{a}}$, Lewis Waller ${ }^{\mathrm{a}}$, Denis Whittard ${ }^{\mathrm{a}}$, Elizabeth Wylie de Boer ${ }^{\mathrm{b}}$, Pascal Xavier ${ }^{\mathrm{a}}$, \\ Jessica Zheng ${ }^{\mathrm{a}}$, Ross Zhelem ${ }^{\mathrm{a}}$, Daniel Zucker ${ }^{\mathrm{a}, \mathrm{g}}$ \\ ${ }^{a}$ Australian Astronomical Observatory (AAO) PO Box 296, Epping NSW 1710, Australia \\ ${ }^{\mathrm{b}}$ Australian National University (ANU), Research School of Astronomy \& Astrophysics, Mount \\ Stromlo Observatory, Weston ACT 2611, Australia \\ ${ }^{\mathrm{c}}$ National Solar Observatory, PO Box 62, Sunspot, NM 88349-0062 \\ d School of Physics, The University of Sydney NSW 2006, Australia; \\ e Prime Optics, Eumundi, QLD 4562, Australia \\ ${ }^{\mathrm{f}}$ Centre for Astrophysics Research (CAR), School of Physics, Astronomy and Mathematics, \\ University of Hertfordshire (Herts), Hatfield, AL10 9AB, UK \\ g Department of Physics and Astronomy, Macquarie University, NSW 2109, Australia
}

\begin{abstract}
The High Efficiency and Resolution Multi Element Spectrograph, HERMES is an optical spectrograph designed primarily for the GALAH, Galactic Archeology Survey, the first major attempt to create a detailed understanding of galaxy formation and evolution by studying the history of our own galaxy, the Milky Way ${ }^{1}$. The goal of the GALAH survey is to reconstruct the mass assembly history of the of the Milky way, through a detailed spatially tagged abundance study of one million stars in the Milky Way. The spectrograph will be based at the Anglo Australian Telescope (AAT) and be fed with the existing $2 \mathrm{dF}$ robotic fibre positioning system. The spectrograph uses VPH-gratings to achieve a spectral resolving power of 28,000 in standard mode and also provides a high resolution mode ranging between 40,000 to 50,000 using a slit mask. The GALAH survey requires a SNR greater than 100 aiming for a star brightness of $V=14$. The total spectral coverage of the four channels is about $100 \mathrm{~nm}$ between 370 and $1000 \mathrm{~nm}$ for up to 392 simultaneous targets within the 2 degree field of view.

Current efforts are focused on manufacturing and integration. The delivery date of spectrograph at the telescope is scheduled for 2013. A performance prediction is presented and a complete overview of the status of the HERMES spectrograph is given. This paper details the following specific topics:

The approach to AIT, the manufacturing and integration of the large mechanical frame, the opto-mechanical slit assembly, collimator optics and cameras, VPH gratings, cryostats, fibre cable assembly, instrument control hardware and software, data reduction.
\end{abstract}

Keywords: HERMES, spectrograph, AAT, 2dF, VPH, GALAH, fibre

*asheinis@aao.gov.au; phone +61 2 93724821; fax +61 2 93724880; www.aao.gov.au

Ground-based and Airborne Instrumentation for Astronomy IV, edited by lan S. McLean, Suzanne K. Ramsay, Hideki Takami, Proc. of SPIE Vol. 8446, 84460W · @ 2012 SPIE · CCC code: 0277-786X/12/\$18 · doi: 10.1117/12.925806 


\section{INTRODUCTION}

In May 2009 the Australian astronomical community agreed that a dedicated survey instrument should be built for the Anglo-Australian Telescope (AAT) that enables chemical tagging of stars in our Milky way. This science is known as Galactic Archeology. Four essential wavelength bands have been identified that must be covered by the spectrograph. HERMES should provide a nominal spectral resolving power of 28,000 for Galactic Archaeology with a high-resolution mode of about 45,000. The opto-mechanical design of HERMES ${ }^{2}$ allows for reconfiguration of the bands to enable astronomers to pursue other spectral bands of interest between $370-1000 \mathrm{~nm}$. The spectrograph will use four large $500 \mathrm{x}$ 200mm Volume Phase Holographic (VPH) gratings.

HERMES is fed by fibres that are positioned by a robot called $2 \mathrm{dF}$ at the AAT telescope prime focus. There are a total of 784 science fibres, which interface with the spectrograph via two separate slits located at the entrance of the spectrograph, each comprising of 392 science fibres.

The HERMES spectrograph has completed its final design and is currently undergoing assembly, integration and testing at the AAO head quarters in Sydney.

\section{APPROACH TO ASSEMBLY, INTEGRATION AND TESTING}

The approach used for assembly, integration and testing is in accordance with the AAO System Engineering Manual. The manual is based on the ISO standard (ISO/IEC 15288 systems and software engineering - system life cycle processes) and the best practice INCOSE systems engineering handbook.

The process is defined to ensure the delivered HERMES system satisfies the stakeholder requirements. The input is the high-level user requirements and science requirements, which flow into the functional and performance requirements document at the next level down. An architectural design has been produced defining the system elements and interfaces (including human elements and external systems). The requirements are then allocated to the subsystem specifications to design and implement each individual element.

This development process was reviewed at the project phases of conceptual, preliminary, and final design. Once the technical development process was completed, the production of the system began and system elements were progressively integrated together based on the architectural design and the system integration strategy. Verification occurs throughout this integration process to identify and rectifying problems early in the process. The process follows the classical V-Model for systems engineering.

Manufacturing specifications that have been formally reviewed are in place for all HERMES components sourced from external suppliers. These specifications are used to procure mechanical components, electrical components and optical components. The specifications are also used as a check list of parameters to verify built components against and identify problems.

Quality Assurance has been carried out throughout the project in accordance with the AAO Quality Manual. The AAO Quality Manual is based on ISO 9001. All parts are independently checked for conformance to specification once they are received. All test results are recorded in the AAO document management system. As each of the sub-systems are integrated (camera, collimator, slit, cryostats, etc.), verification occurs against the sub-system specifications. Non conformance reports are raised for any parts that do not meet specification.

The system integration strategy is to first fully integrate and test the blue channel of the instrument. This strategy will identify any issues in the instrument early as well as to produce and reduce a spectrum. The information gathered could also be used to rectify problems in the green, red and infrared channels before they are fully assembled.

The blue channel of HERMES will be tested against the functional and performance requirements document at the AAO laboratories. Test fibres will be used to simulate the AAT fibre feed and obtain fully reduced spectra.

After the blue channel testing is completed, the remaining three channels will be built and tested. The spectrograph will then be transported to the AAT where it will be re-tested against the functional and performance requirements. The full HERMES system will then be commissioned against the user and science requirements. 


\section{OPTICAL COMPONENTS}

The majority of the optical components are fairly large in size, in the order of half-a-meter, except for the small slit relay optics (5mm lenses) and the large collimator mirror (1meter). This means that in general terms the production times are long, the development is expensive, and the number of available suppliers is small. In the next paragraphs the status of the optical components for the HERMES spectrograph as in June 2012 are presented.

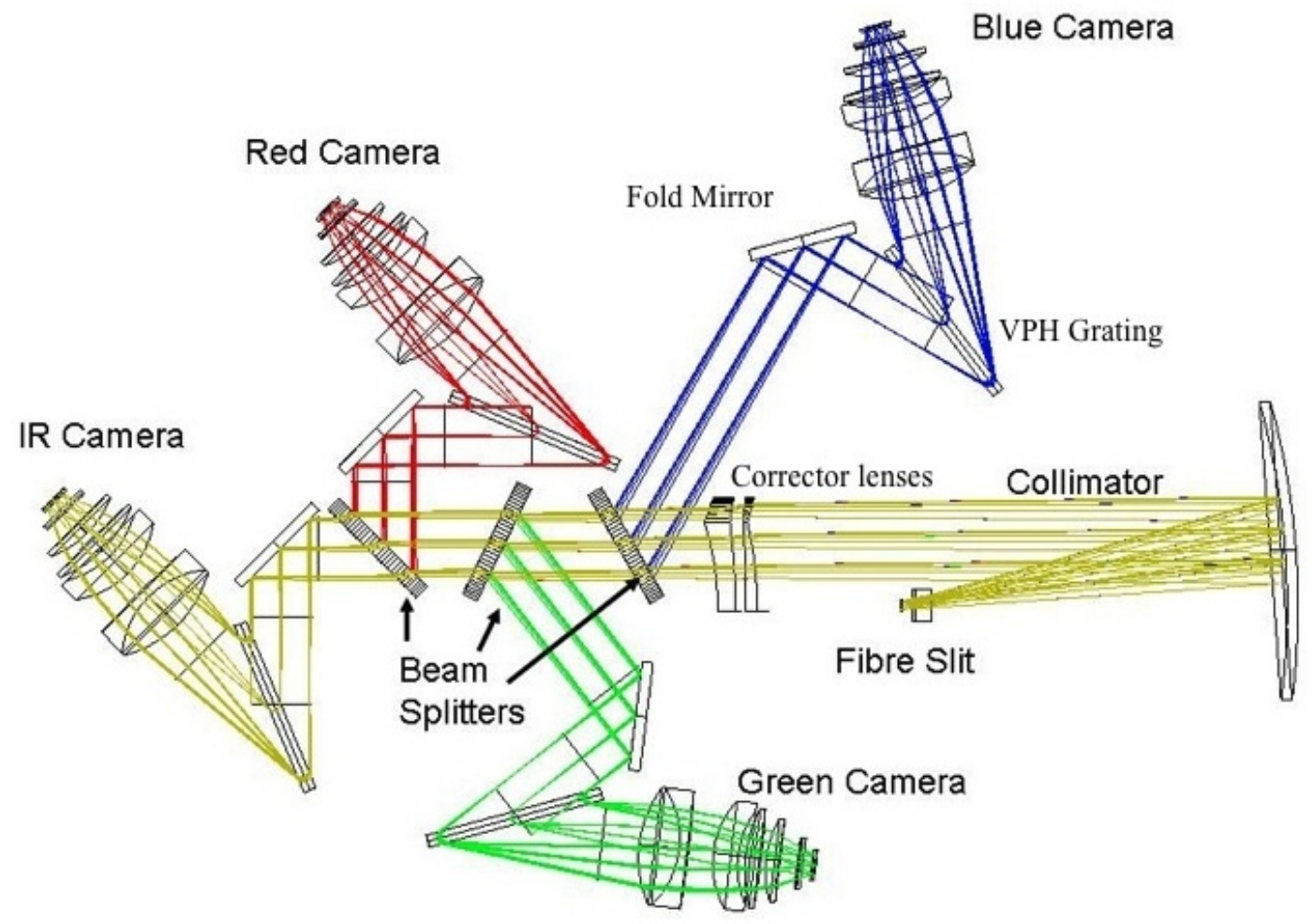

Figure 1. Optical layout of the HERMES spectrograph

\subsection{Slit relay optics}

All 80 sets of slit relay lenses have been manufactured within its high dimensional tolerances (several micrometers) ${ }^{3}$. Each set consist of three lenses that reimage the light from 10 fibres with a focal ratio of F/3.15 into F/6.3. The lenses are assembled in a block with V-grooves that create a spherical curved slit. Figure 2 shows the realized slit assembly.

\subsection{Collimator assembly}

The collimator is an off-axis F/6.3 Houghton design with a spherical mirror and two spherical corrector lenses. The mirror, made from Astrocitel, has been completed. The mirror is chain mounted and its mounted wave front quality is close to $1 / 10$ wave over its effective aperture of 0.91 meter.

The enhanced Silver coating has a reflectivity of more than $95 \%$ over the wavelength band of $470-1000 \mathrm{~nm}$. This turned out to be a challenging development, especially for the lower wavelengths and the required flat response. 


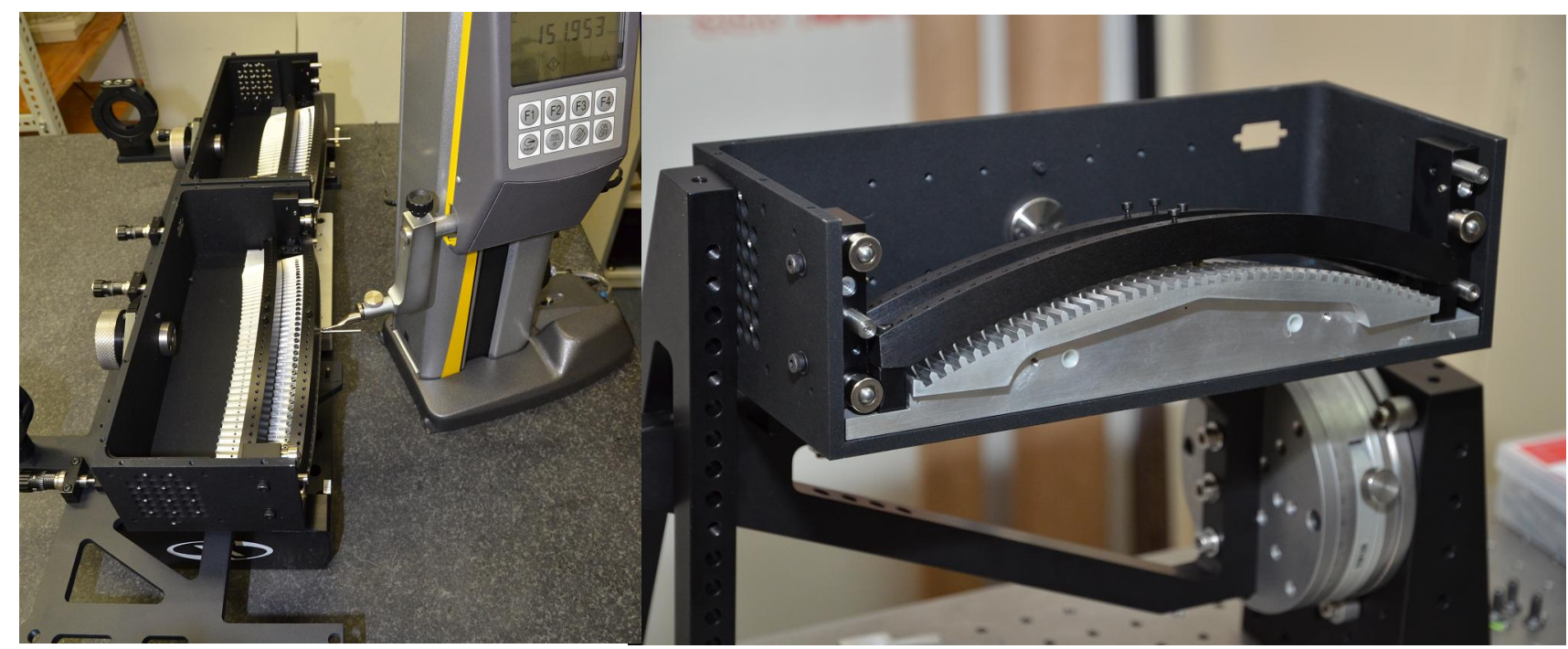

Figure 2. The two curved slit assemblies on the translation stage, right: single V-groove block for lens mounting 40 lens sets
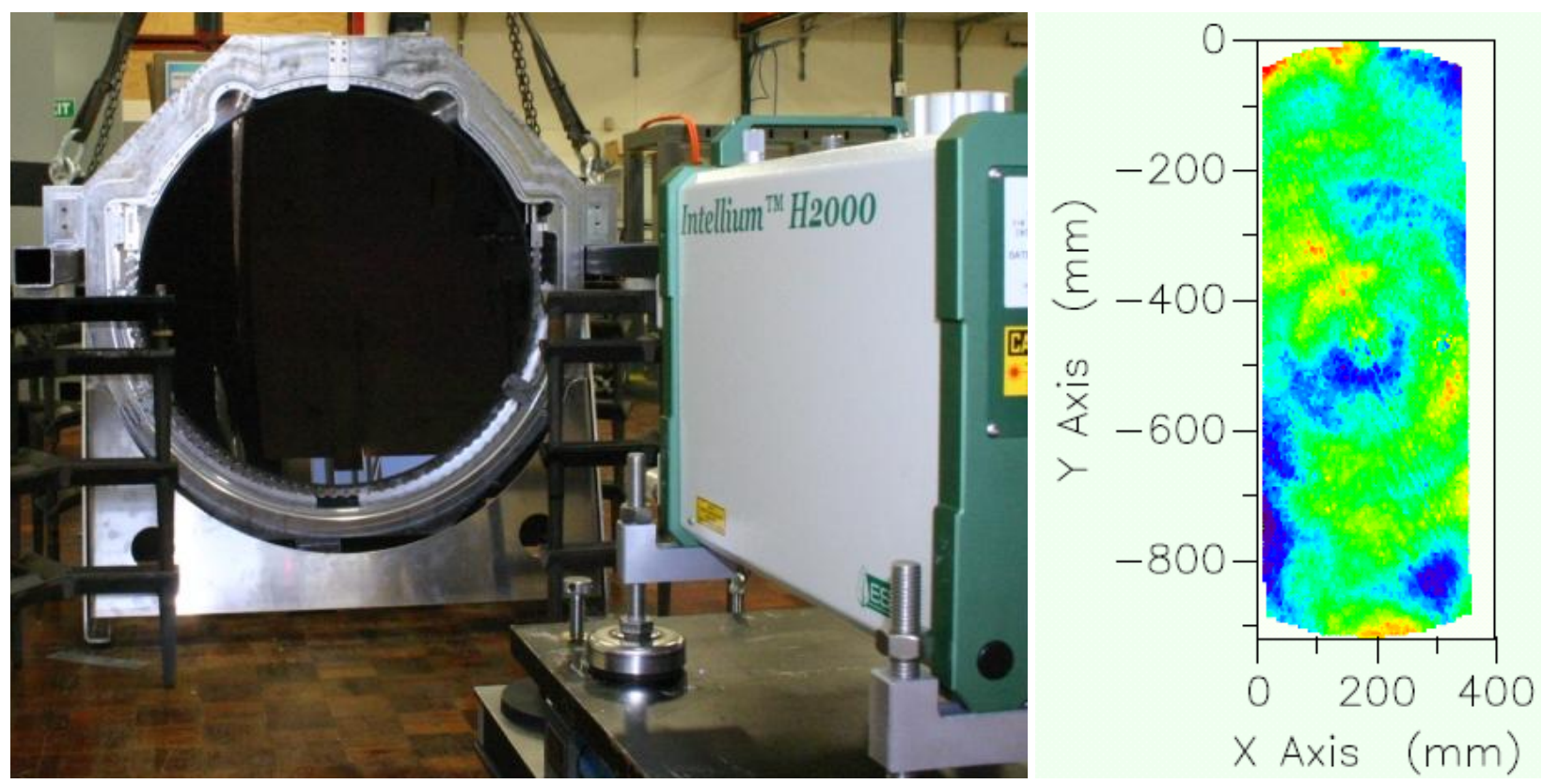

Figure 3.Collimator mirror before coating and its interferogram: $P-V=0.1$ wave, $R M S=0.013$ wave, (blue: -0.05 , red: +0.05 wave)

The two large BK7 collimator corrector lenses, with a maximum height of $620 \mathrm{~mm}$ have been polished and cut to size. The lenses have been cut from their larger circular substrates which introduced an increase in the radius of curvature. The wavefront increased from less than 0.25 waves before cutting to 0.6 waves. After analyses this combined wavefront of the two lenses was analysed and deemed sufficient. 

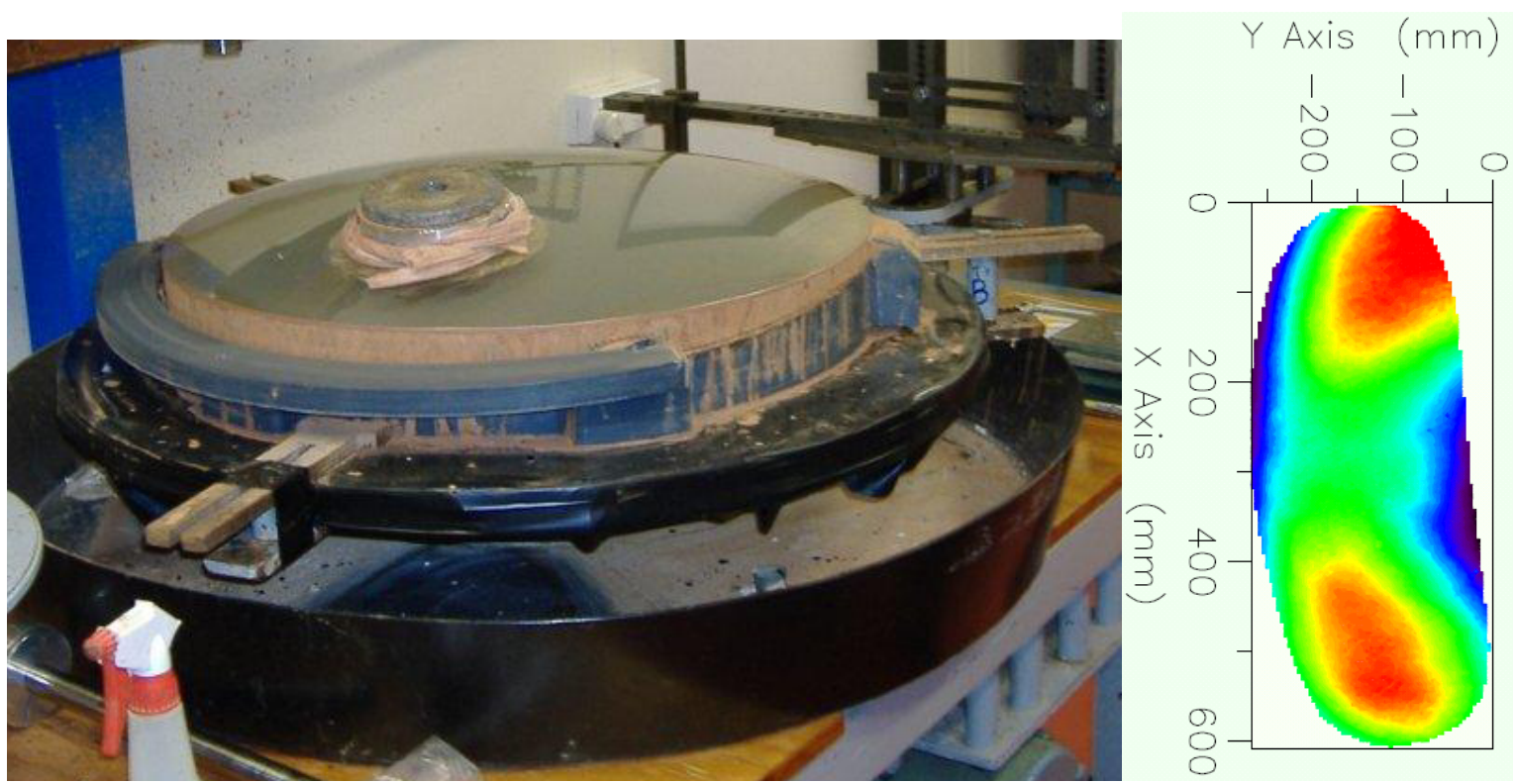

Figure 4. Polishing Collimator lens 1, right: concave surface interferogram: $\mathrm{P}-\mathrm{V}=0.6, \mathrm{RMS}=0.13$ wave (blue:- 0.36 , red: +0.24 wave)

\subsection{Beam splitters}

The beam splitters that define the wavelengths for the four channels are as follows: $370-492 \mathrm{~nm}$; $560-593 \mathrm{~nm}$; 643 $679 \mathrm{~nm} ; 754-1000 \mathrm{~nm}$. The dimensions of the BK7 substrates are 400x 530mm with a thickness of 50mm. After the first polishing attempt the beam splitters did not meet the required flatness of $1 / 6$ wave over a quarter of the aperture. A second attempt with double sided polishing gave an excellent flatness of 1/10 of a wave over the full aperture. However, concerns were raised about introduction of bending stress caused by the dichroic coating on one side and an AR coating on the other. After testing, the predicted bending radius for a full sized, single sided dichroic coating is $200 \mathrm{~km}$, or half a wave deflection. Analysis showed that this power term would not cause significant image quality degradation. The blue beam splitter is currently being prepared for coating.

\subsection{VPH gratings}

HERMES makes use of unique Volume Phase Holographic (VPH) gratings that operate under an extreme angle of 67-68 degrees ${ }^{4}$. Two of the four gratings require a mosaic of two gratings on one substrate due to the aperture and line frequency required. As these gratings are unique this has led to a significant design and research effort. The biggest challenges have been to achieve a grating that would have an efficiency of at least $40 \%$ and a line homogeneity over its full $190 \mathrm{~mm}$ aperture of $2 \mathrm{e}^{-6}$.

The first grating that has been produced is the blue grating that operates over $470-490 \mathrm{~nm}$. The measured efficiency, corrected for reflection losses is $40 \%$ at the edge and $49 \%$ at the central wavelength. This grating is scheduled to be AR coated in July 2012 and should arrive at the AAO in the same month. The coating is optimised for S-polarisation and has been extensively tested. The coating is applied in a low temperature process of less than $100^{\circ} \mathrm{C}$ to prevent the grating gelatin from damaging. 


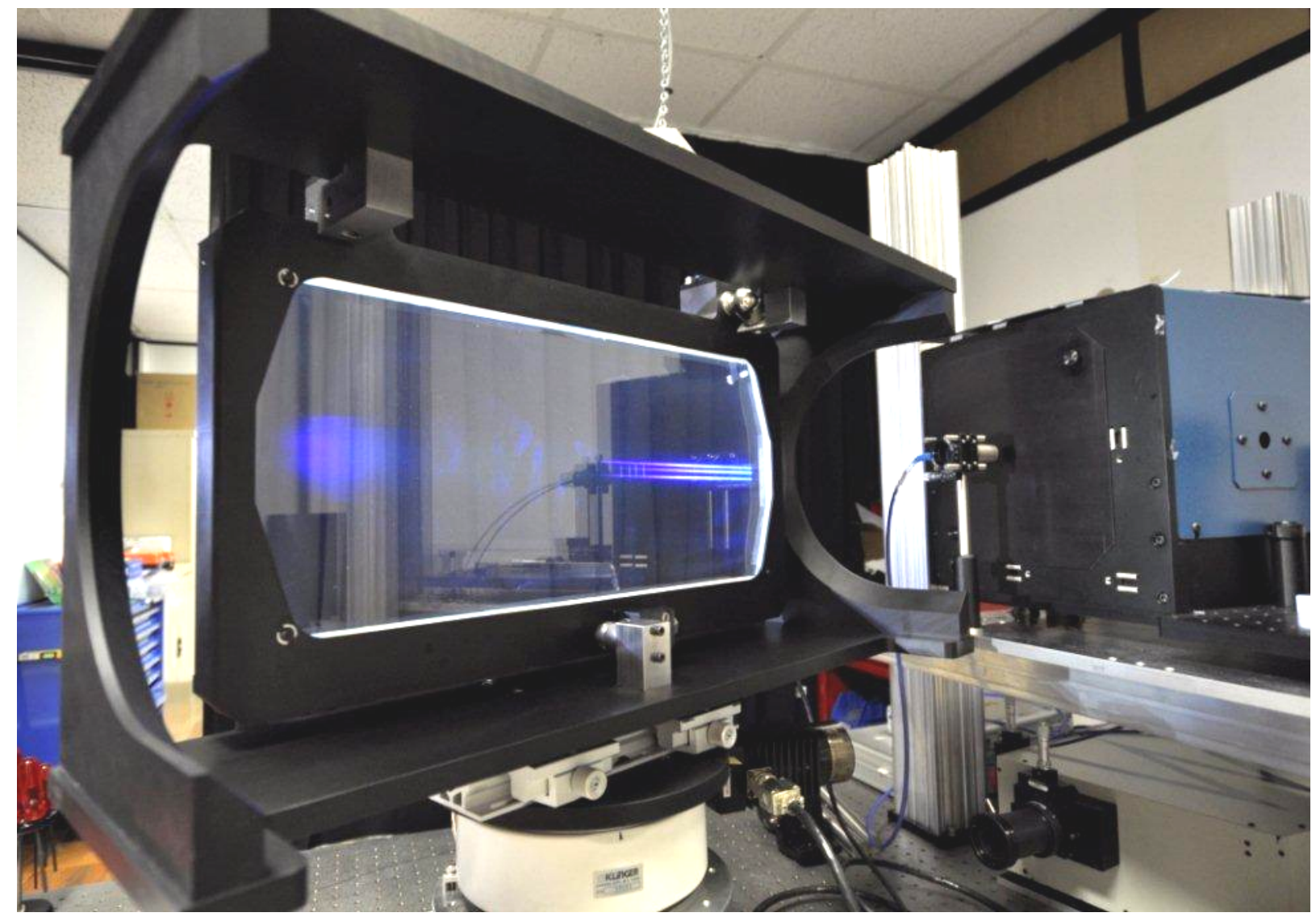

Figure 5. The first test run of the full sized $240 \times 570 \mathrm{~mm}$ blue grating

The green grating has a central wavelength of $578.8 \mathrm{~nm}$ and is also a single exposure grating. This grating is currently under manufacture. The red and IR gratings are expected to arrive late this year and have a central wavelength of $664.2 \mathrm{~nm}$ and $777.8 \mathrm{~nm}$ respectively. The latter two have a double mosaic.

\subsection{Cameras}

The cameras are fairly fast with an F number of 1.7. The largest elements are the doublets with a diameter of $380 \mathrm{~mm}$. All four camera lenses have been polished and at the moment of writing this publication the blue and green camera lenses have been AR coated. The coatings for the red and infra red cameras are scheduled to be completed mid 2012.

Manufacturing of the lenses has introduced a significant delay with a wide range of causes from damaged surfaces, breakage and scheduling problems between suppliers.

Some of the large lenses have a strong curvature that makes coating a non trivial process. The four cameras operate between $370 \mathrm{~nm}$ and $1000 \mathrm{~nm}$ with some overlap. The lens design of the red and IR cameras is the same but these have different lens spacings and different coatings. The designed and measured reflectance of the blue AR coated lens surface is less than $1 \%$.

A suitable RTV adhesive has been found and tested and assembly of the blue camera is scheduled to be completed by July 2012.

The field flatteners that sit on top of the detectors with a four millimeter separation have been coated with a coating that is suitable for cryogenic use. 


\section{THE MECHANICAL FRAME}

The frame of HERMES positions the optical components with a high geometric stability. The frame creates a stiff support structure which then rests on four vibration isolators. The vibration isolators provide decoupling of the rigid structure from building vibration. The material chosen for this structure is Alcoa MIC-6 aluminum tooling plate of .5 inch, $.75 \mathrm{inch}$, and 1 inch thickness. Due to its unique casting process and being fully stress relieved this material provides a high degree of geometric stability post machining. Using an aluminum alloy provides excellent thermal behavior since it has a high thermal capacity and a high thermal conductivity. The frame has a high thermal inertia allowing it to resist room temperature variation while the high thermal conductivity allows for uniformity of temperature across the structure. This HERMES Spectrograph will operate in a thermally insulated room that is tightly temperature controlled. The room design and HERMES frame design will provide a temporally stable optical alignment.

This first integration of HERMES occurs in Epping-Sydney and after successful testing the instrument will be shipped to the telescope in Siding Springs, NSW. This plan necessitated designing the HERMES frame as a modular unit. The frame design has a central support structure created out of 1 inch MIC-6 plate over $3 \mathrm{~m}$ long. This main support structure is called the "spine" of HERMES. The spine supports the slit assembly, field lens, collimator mirror, corrector lenses, and beam splitters. Off the spine are four structures that are bolted and pinned to the spine. Each of these structures is for one arm of the spectrograph. These structures support a grating, fold mirror, and light baffling. Cantilevered off each of the four channel structures, is the camera focus drive assemblies and cryostat assemblies.

Utilizing the MIC-6 plate provided the opportunity for a simplified manufacturing process. MIC-6 plate is received ground on each face which leaves them very flat with an excellent surface finish. No surface milling was performed on this design. The plates were water jet cut from sheet stock to rough size. The AAO provided cut sheets to the water jet cutter to optimize material usage and minimize waste. After roughing, the sheets were sent to various local manufacturers for machining. The machining process mostly entailed profiling the rough edge of the plate and then various hole drilling processes on the surface. This method minimized machining cost for the program and allowed for a high quality finished product as machining done in this fashion allows for a very high accuracy. The position accuracies of most of the optical assemblies are set by spacers or shims that are machined to size. The angles for the reflective and diffractive components are set by micrometer adjustors in combination with a kinematic mounts.

The HERMES frame has been fully assembled. No significant issues were encountered during the initial build up. Figure 6 shows the structure which supports the collimator mirror and the blue channel mounting area. Figure 7 shows the structure including the mounting area for the red and IR channels 


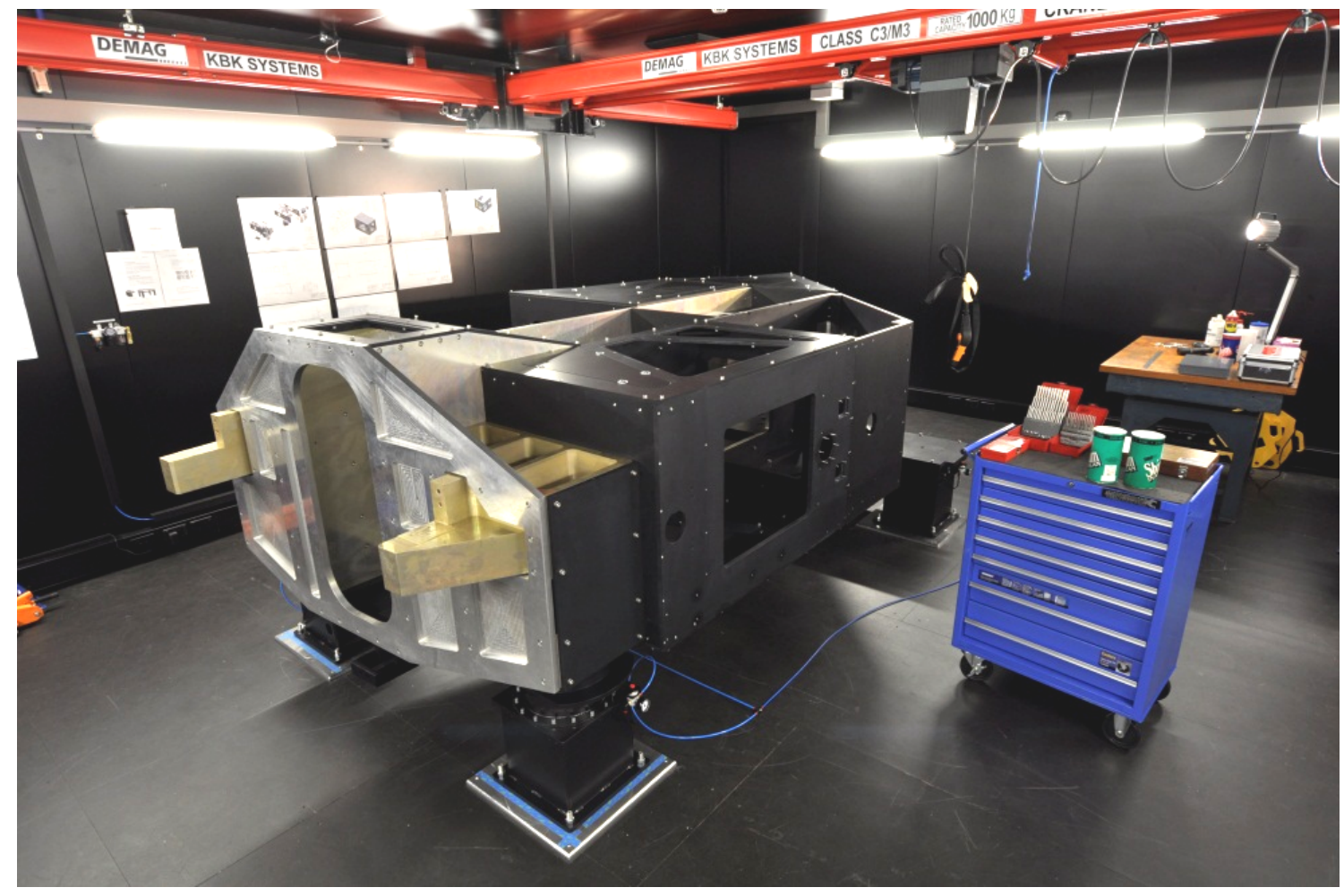

Figure 6. The HERMES spectrograph frame as seen from the collimator mirror support

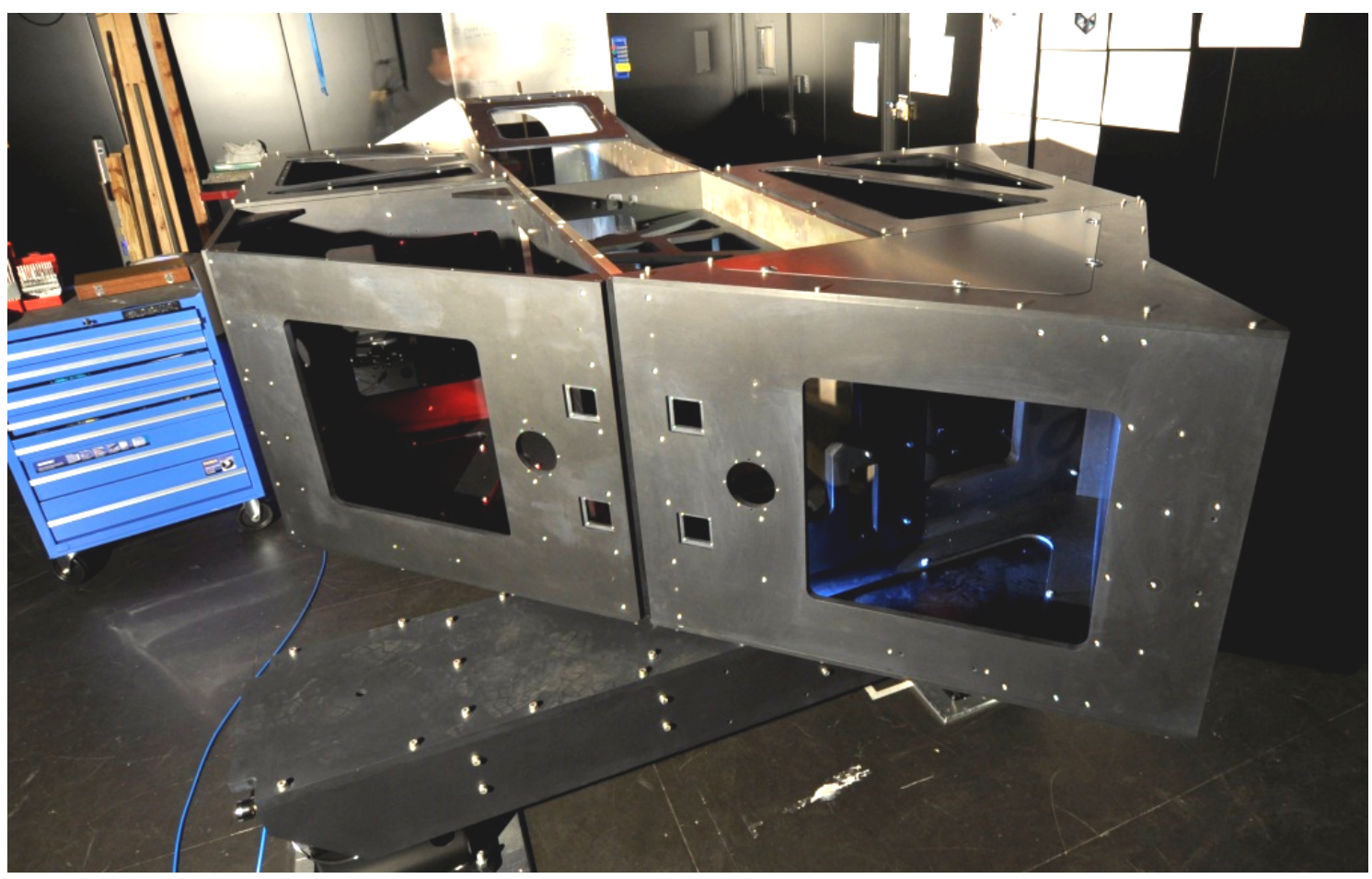

Figure 7. The HERMES spectrograph frame as seen from the red and IR channel mounting area 


\section{CRYOSTATS AND DETECTORS}

The HERMES detector cryostat shell is based on an Infrared Laboratories cryostat with a Polycold PCC cryocooler cold head to which we have fitted a Pfeiffer PKR full range vacuum gauge head. The end plate is replaced by a custom shell extension which encloses the detector and field flattener assembly. Hermetically sealed connectors, one for the detector control and data, another for thermal control, are mounted through the wall of this shell extension. To maintain thermal isolation a G10 fibreglass 'spider' is used to locate the detector and field flattener to the wall.

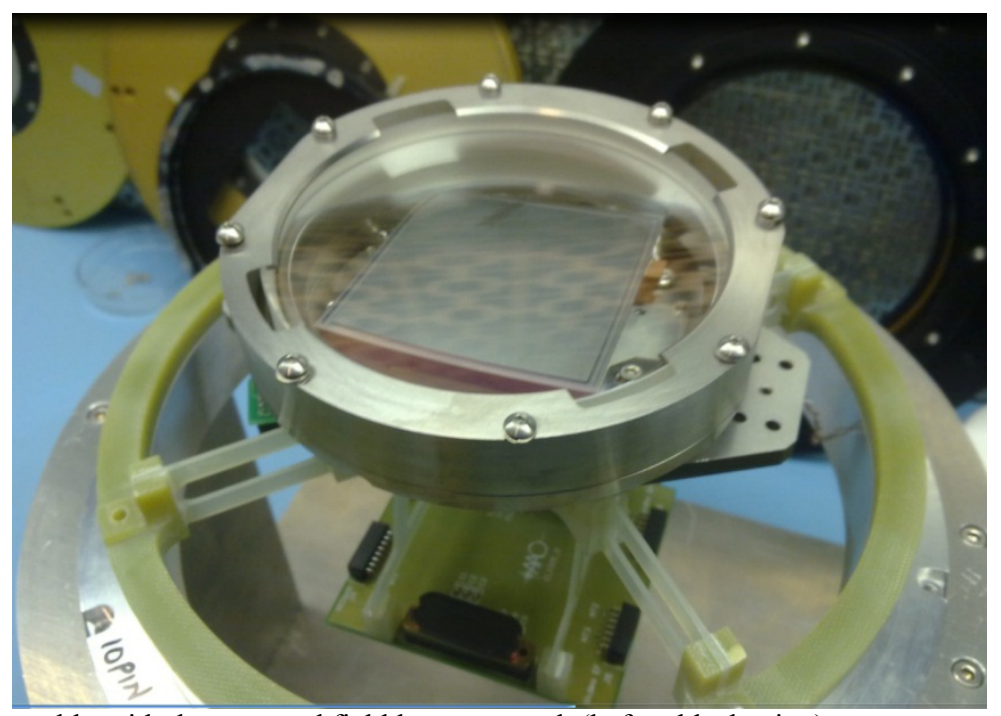

Figure 8 . The detector assembly with the mounted field lens mounted, (before blackening)

To ensure a good match to the thermal expansion of the CCD detector, a molybdenum mounting plate is used. The thermal control hardware, heaters and a diode sensor are mounted directly on this plate. Three heaters are used to provide an even distribution of the heat input required for temperature trimming to much less than $0.1^{\circ} \mathrm{C}$.

The mount for the bi-concave field flattener lens is mounted with less than $4 \mathrm{~mm}$ gap from the detector. Cooling of the detector assembly is implemented by linking the detector mounting plate to the cryocooler head using oxygen-free copper thermal links. To minimize thermal strains and maintain detector alignment these links incorporate corrugated sections which structurally decouple the cryocooler head from the detector assembly. The contamination 'getter' is also connected via separate links to the cold head. A radiation shield encloses the detector and field flattener assembly and the thermal link and getter assembly. The cryostat shell is sealed by a front plate which accommodates the plane window, which withstands the atmospheric pressure load and a second thinner window. Dry nitrogen may be flushed between these windows to prevent dewing that might otherwise occur on high-humidity nights.

Internal electrical wiring is accomplished using flexible PCBs and the detector controller electronics box is mounted directly on the cryostat shell to minimize noise.

Design variations between cryostats result from the different wavelength ranges at which they operate and are limited to different CCDs, field flatteners and window coatings.

The detector control electronics includes the CCD controllers and their power supplies for each detector, as well as the computer systems that send commands to and acquire data from the CCD controllers. Each CCD detector in HERMES is controlled with an AAO2 CCD controller. ${ }^{5}$ These controllers are configured for operation with the e2v CCD231-84 detectors to permit readout from one, two or four detector outputs, at various readout rates with windowing and binning. The CCD controller electronics and enclosures have been manufactured and most of the individual boards have been tested. Three of the four HERMES CCDs have been tested and characterised in a test cryostat and the "engineering" grade detector has been installed in the blue camera cryostat and is undergoing performance verification tests prior to the blue science grade detector being installed. At this point the blue cryostat is showing excellent vacuum and thermal performance. The next step will be to swap the blue science grade detector into the cryostat. 


\section{FIBRE CABLE FEED}

The HERMES/AAOmega fibre bundle was designed to co-inhabit the $2 \mathrm{dF}$ robot positioner so that no instrument changeovers were required to switch between HERMES and AAOmega, also no need for connectorisation ${ }^{6}$. This led to the design of two fibres occupying one magnetic button, one for AAOmega fibre and one HERMES fibre, see the figure below. The $2 \mathrm{dF}$ robot positions the magnetic button on the field plate and determines its position by looking at the back illuminated science fibre. .

The fibre cable, with its $4 \times 400$ fibres, has been built and equipped with its slit and button assembly. Delays have been experienced due to magnet strength issues and prism quality issues. At this stage the HERMES \& AAOmega fibre cables have been assembled with their protective conduit in preparation for its installation on $2 \mathrm{dF}$ in October this year when $2 \mathrm{dF}$ is scheduled to be off the telescope.

The existing fibre cable had a fringing problem that caused a wavelength-dependent intensity fluctuation in the measured spectra. This was found to be related with a cavity that was formed between the prism and the fibre face at the button end of the fibre cable. This connection has been redesigned and adhesives are used that have a better thermal match, low shrinkage and creates a strong bond. The assembly was tested on the AAT for five months and no fringing was observed.

Another point of attention has been to prevent the fibres from stressing as this can cause focal ratio degradation, (FRD). With a fibre cable of almost 50 meters in length there are many points where the fibre is bent and fixated. With large bending radii, protective sleeves and low stress points at fixations, the FRD was kept low and the achieved throughput for the new fibre cable is significantly higher $(>50 \%)$ than the existing cable.

The $2 \mathrm{dF}$ buttons comprise of fibres terminated in a glass ferrule with a polyimide jacket covering the two AAOmega/HERMES science fibres plus a short ( $30 \mathrm{~mm} \mathrm{long)} \mathrm{dummy} \mathrm{fibre} \mathrm{to} \mathrm{align} \mathrm{the} \mathrm{fibres} \mathrm{perpendicular} \mathrm{to} \mathrm{the} \mathrm{prism}$ face. These ferrules are then glued into magnetic buttons to locate them on the $2 \mathrm{dF}$ field plate. The images below show the assembly.

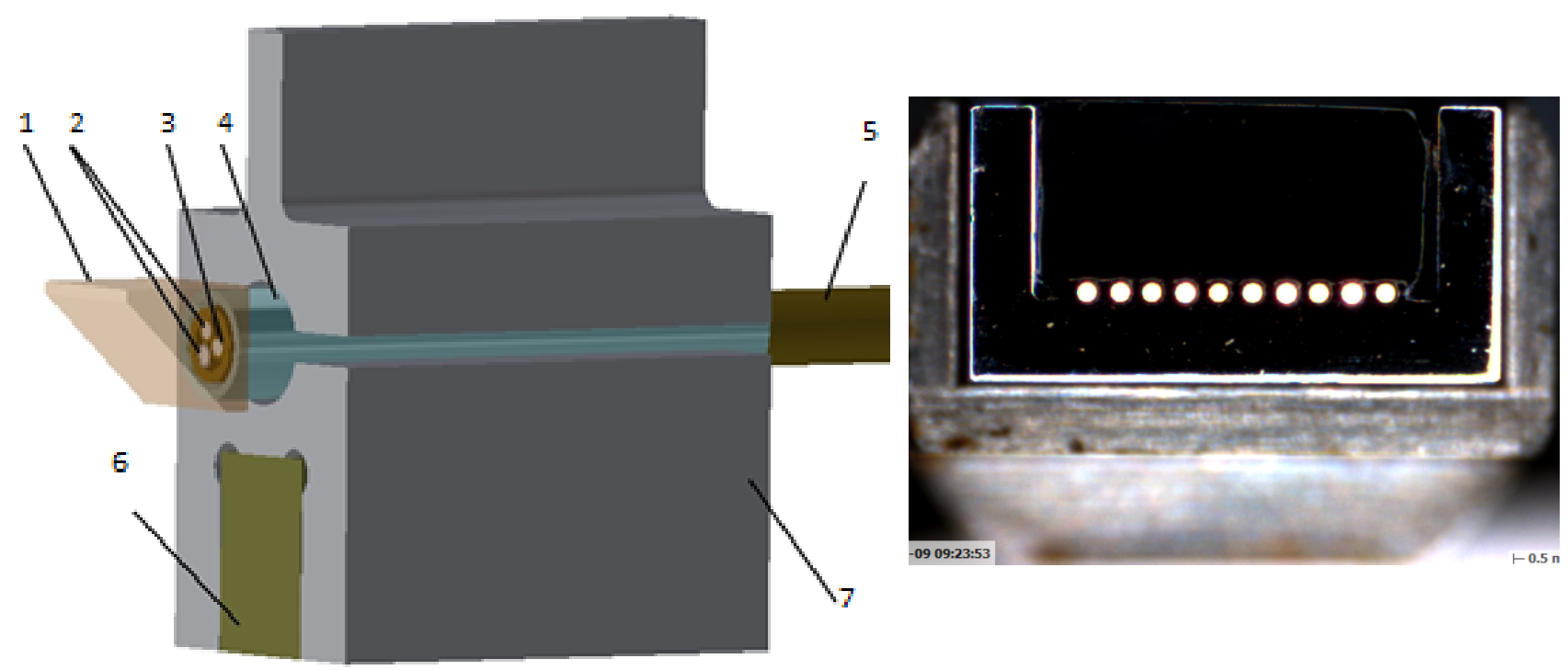

Figure 9. 2dF button. 1- Prism. 2- AAOmega/HERMES science fibre. 3- Dummy fibre. 4- Glass ferrule. 5- Polyimide tube. 6 Magnet. 7- Button body (Prism size is $1 \mathrm{~mm}$ ). Right: an image of 10 fibres in a slitlet

At the slit entrance the fibres are assembled in slitlets that locate 10 fibres in v-grooves and collectively they make up a slit of 400 fibres.

The HERMES slitlets were glued and polished successfully. The locations of the fibres in their v-grooves with respect to their nominal positions relative to the slitlet body were measured using a MicroVu co-ordinate measurement system. The fibre locations were within the required radial position tolerance of $+/-10 \mu \mathrm{m}$ to prevent crosstalk at the detector images. 


\section{CONTROLLERS AND ELECTRONICS}

The electronics for HERMES is broadly separated into two categories: instrument control electronics and detector control electronics, as shown in Figure 10. The HERMES instrument control electronics is responsible for controlling and monitoring all the actuators and sensors used to configure and operate the instrument. The instrument control electronics is based on a distributed control system that uses the CANopen protocol running on the Controller Area Network (CAN) field bus. All analog and digital functions are interfaced to CANopen input/output nodes and all servo motor driven actuators are controlled by CANopen digital servo drives. The CAN bus is connected to an industrial control cabinet PC with a CAN interface, running the Linux operating system and operating as the CANopen master. The instrument control electronics is implemented in six industrial electronics enclosures distributed around the outside of the spectrograph thermal enclosure. The majority of the instrument control electronics is commercial off-the-shelf. Some customization has been done on the slit back illumination control and drive, interlocking and interfacing for the Bonn shutters and servo amplifier breakout.

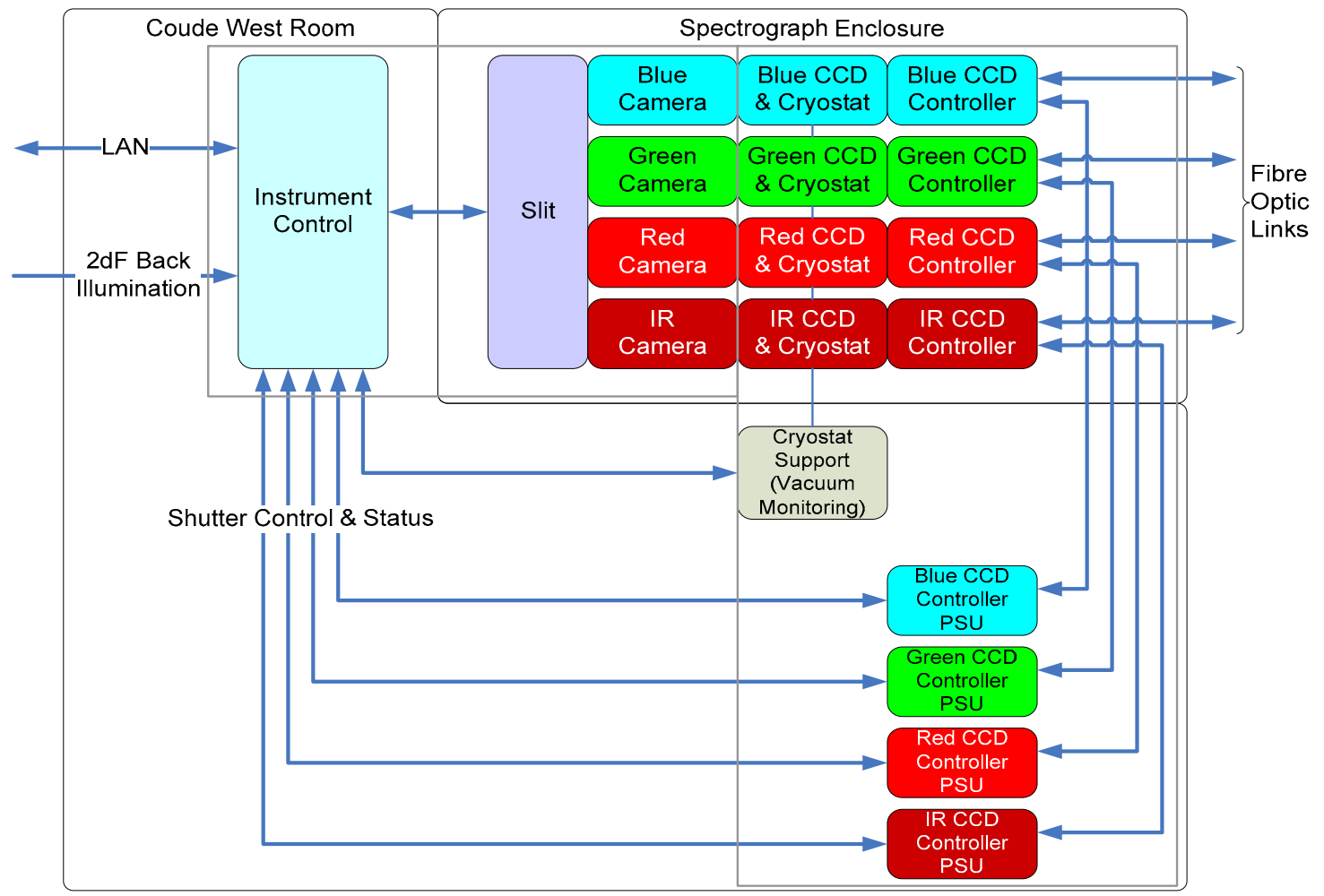

Figure 10. Schematic overview of the HERMES Electronics Systems

A CAN/CANopen system including a digital servo drive and digital and analog input and output was prototyped during the final design phase of the project. This has been available to allow software and hardware development and testing to proceed from an early stage in the project.

The assembly of the instrument control electronics is largely complete, and is currently undergoing power up and basic functionality tests prior to being integrated with the instrument hardware. 


\section{SOFTWARE AND DATA REDUCTION}

\subsection{Architecture and layout}

Extensive use is being made of existing software deployed as part of the 2dF/AAOmega instrument. This software was written using the AAO's DRAMA API ${ }^{7}$ for the deployment of the original 2dF Instrument configuration, in 1994, but has been modified over the years for new spectrographs, detector systems and configurations.

For development purposes, the software for HERMES is broken into five major areas. The Observing system is responsible for overall control of the entire observing run-time (including the $2 \mathrm{dF}$ robot and CCD operations) and provides the main GUI. The HERMES Spectrograph Control task is responsible for control of the spectrograph itself. The AAO2 CCD detector software ${ }^{8}$, shown in the figure below as CCD System, is used to run the detector systems. The Fibre Configuration software, not shown, is responsible for allocating fibres to objects as part of proposal preparation. A separate Data Reduction package provides pipeline reduction to the point of producing calibrated spectra.

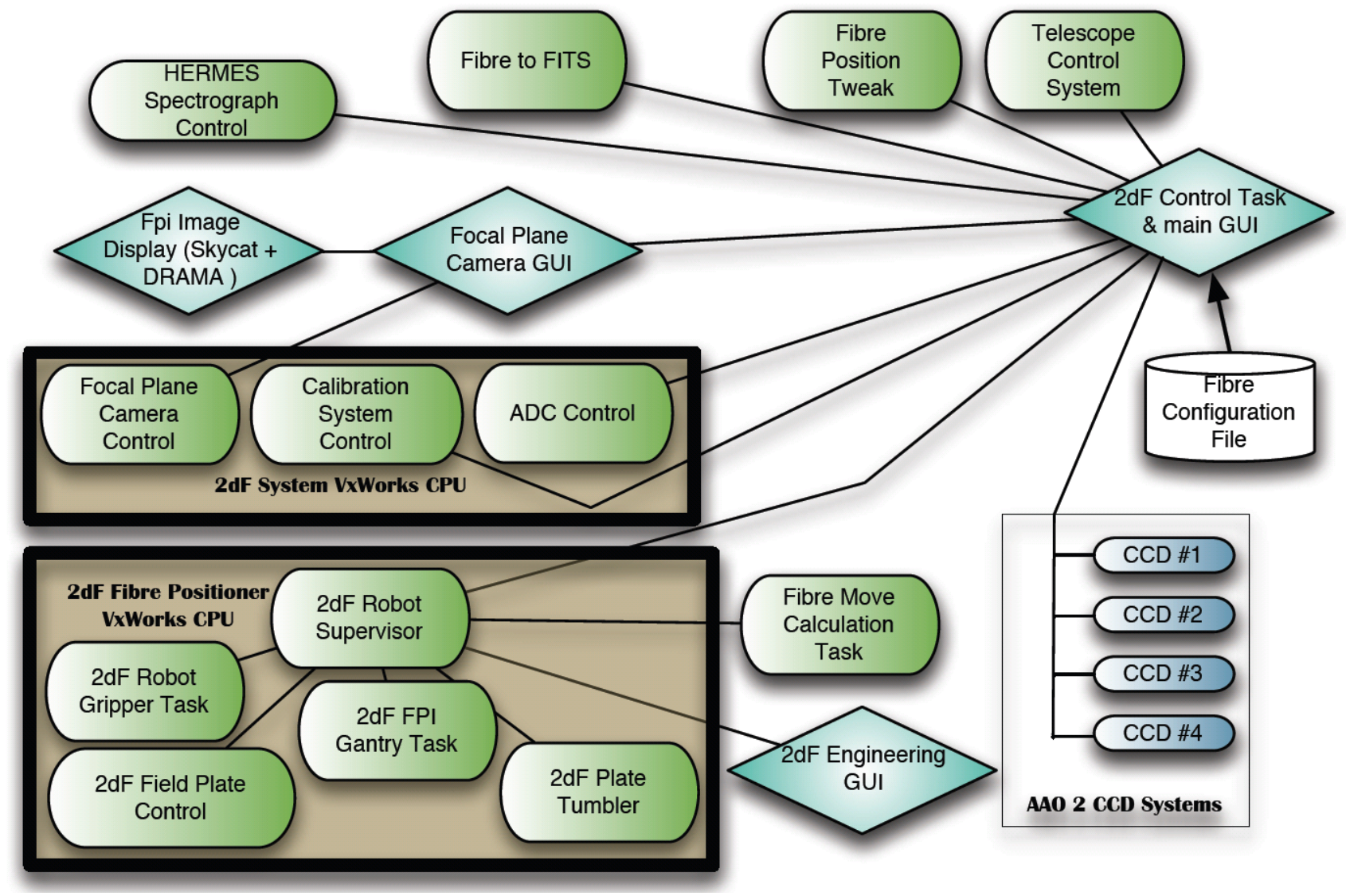

Figure 11. Overview of the $2 \mathrm{dF}$ observing system software tasks when running in HERMES mode

\subsection{The HERMES Observing System}

The existing $2 \mathrm{dF}$ Observing system has been modified to control HERMES in addition to AAOmega. The Observing system controls the 2dF Fibre Positioner, the Spectrographs, Detector Systems, 2dF Calibration system, 2dF Atmospheric Dispersion Corrector, the Telescope and a number of minor components. Each sub-system has its own DRAMA task. The AAO 2 CCD detector software is itself an independent set of DRAMA tasks used by the $2 \mathrm{dF}$ software, as is the AAT's Telescope Control software. Overall control, software interlocking and synchronization are provided by the $2 \mathrm{dF}$ Control Task. This Observing system has proven flexible and adaptable since being originally commissioned in 1994, with the CCD System, Telescope System and Spectrographs having been changed during that time. For HERMES, we have adapted the control task to implement a new mode of operation where the AAOmega 
Spectrograph DRAMA task is replaced by the new HERMES Spectrograph DRAMA task. Additionally, the control task and detector software were adapted to control four CCD systems, rather than the two previously supported. Switching between AAOmega mode and HERMES mode is via a command line option at system startup.

All sub-systems of the $2 \mathrm{dF}$ Observing system are required to implement simulation modes that allow for development of the Observing System without access to the instrument hardware. This has been used extensively and as a result the $2 \mathrm{dF}$ Control Task has been largely adapted to work with HERMES. All major work is complete and the system could be deployed at this point in time. A significant number of minor changes intended to improve the efficiency of the system remain to be implemented, but these changes are underway.

\subsection{Spectrograph Control}

A HERMES Spectrograph DRAMA task is required to replace the AAOmega spectrograph task when $2 \mathrm{dF}$ is running in HERMES mode. This task is responsible for communication with the spectrograph hardware and implementing highlevel commands (DRAMA commands) for controlling the hardware. It provides instrument state for inclusion in FITS file headers and makes status information available via DRAMA parameters for inclusion in the system GUI.

An extensive hardware simulator has been provided using instrument simulator technology previously developed at the $\mathrm{AAO}^{9}$. This provides simulation of the CANBUS electronics hardware of the spectrograph at a level that requires no changes to the Spectrograph Task when running in simulation. Individual components of the instrument may be simulated at will. We have used this extensively to advance development of the Spectrograph Task, with the command and parameter interface being complete. Standalone testing of the slit exchange mechanism has been completed and similar testing is currently underway for a detector focus mechanism.

\subsection{CCD Control}

HERMES will be the first AAO Instrument using 4 readouts (quadrants) per detector. It is also the first instrument using 4 detectors at once and using large detectors of $4 \mathrm{kx} 4 \mathrm{k}$ pixels. Previous instruments were limited to 2 readouts per detector and 2 detectors at once. The AAO 2 CCD software was designed to support 4 readouts, but with no such detector configuration previously available, the implementation was not completed. With HERMES detectors available for testing, we have now been able to complete this implementation, whilst at the same time optimizing the HERMES detector control modes. Adaption of the software to support 4 detectors running in one configuration was made early in the HERMES project, and we are now regularly running four detectors in simulation whilst adapting the $2 \mathrm{dF}$ control task for HERMES. Three out of the four detectors are operational and the fourth IR detector will be delivered mid 2012. Unfortunately, due to some hardware limitations, we will not have all 4 actual detector controllers available simultaneously before the HERMES instrument is moved to the AAT, so the final testing of the detector control configuration will not be possible until then, but we believe all high-risk areas will have been tested before that point.

\subsection{Fibre Allocation}

To observe a field on $2 \mathrm{dF}$, you must allocate objects to fibres. This is done using a program known as "AAO Configure". Implemented originally for $2 \mathrm{dF}$ in 1994, it was adapted to support the $6 \mathrm{dF}$ Instrument on the UK Schmidt Telescope and FLAMES on the VLT UT2, and to various changes in $2 \mathrm{dF}$ over the years. It was also modified to implement a Simulated Annealing allocation method.

Minor changes will be required for HERMES - basic instrument support and supporting the use of proper motions ${ }^{10}$. Proper motions had been supported in the FLAMES implementation, but had not been implemented with $2 \mathrm{dF}$ until now. It is possible that we will also need to modify the allocation algorithm for HERMES, to optimize for fibre efficiency considerations such as cross talk

\subsection{Data Reduction and Data Simulator}

The AAO will provide software to reduce the image data files produced by HERMES to the extent of generating calibrated spectra with (as far a practical) all instrument signatures removed. Science processing is left to the science teams, but in this case there is a clear requirement to interface efficiently to the "Galactic Archaeology" abundance pipeline. The AAO's existing Fibre Instrument Reduction Package - 2dFdr, is being adapted to support HERMES. This package has previously proven to be adaptable to different Instruments and such adaptations are now relatively easy to implement. The signal to noise requirements on HERMES to carry out "Galactic Archaeology" combined with the high resolution of the instrument lead to a tough set of requirements being imposed on data reduction for HERMES. 
Additionally, the typical delay of 6 to 12 months after instrument commissioning before data reduction is optimal was considered unacceptable. Fortunately, most requirements proposed for HERMES will also improve the reduction of other instruments supported by the package, allowing significant testing before commissioning. Additionally, we have implemented a Data Simulator ${ }^{11,12}$ capable of producing simulated HERMES frame with all known instrument effects accounted for. The Data Simulator allows us to input spectra from stars with known elemental abundances. Our aim during the testing phase, underway at present, is to generate the simulated data files from these spectra, reduce them and run them through the abundance pipeline to ensure we recover the known abundances within the required accuracy for Galactic Archaeology science.

Many of the major changes required for HERMES have already been implemented and tested with AAOmega data. This includes completion of an Optimal Extraction algorithm ${ }^{13}$, Principle Components Analysis (PCA) Sky Subtraction ${ }^{14}$, Improved Wavelength Calibration using an algorithm based on Whale Shark identification ${ }^{15}$ and various minor improvements. We are concerned that PCA Sky Subtraction may not be appropriate for the Solar like stars of the GA Survey and will investigate alternatives such as techniques based on Total Variation Image Reconstruction ${ }^{16}$ as an alternative.

We are currently in the process testing the data flow from Simulator, through the reduction, to the Abundance pipeline. When the actual blue channel is available for testing, we will use that to confirm our Simulation models and to confirm the reduction of Solar spectrums and recovery of Solar elemental abundances.

\section{SPECTROGRAPH PERFORMANCE}

The GALAH survey has set out challenging specifications that can only be met when the individual components meet their design specifications. The required signal to noise level is close to 100 with a 3600 second exposure time for a star of magnitude V14. The predicted performance of the spectrograph is regularly updated for the latest designs, as built geometries and for the measured (coating) performance.

\subsection{Performance indicators}

The first performance indicator is the total efficiency. In the figure below a "waterfall graph" shows the drop in the efficiency from telescope to detector. The VPH grating and the fibre cable show the significant drop in system efficiency. This graph is based on mainly measured efficiencies.

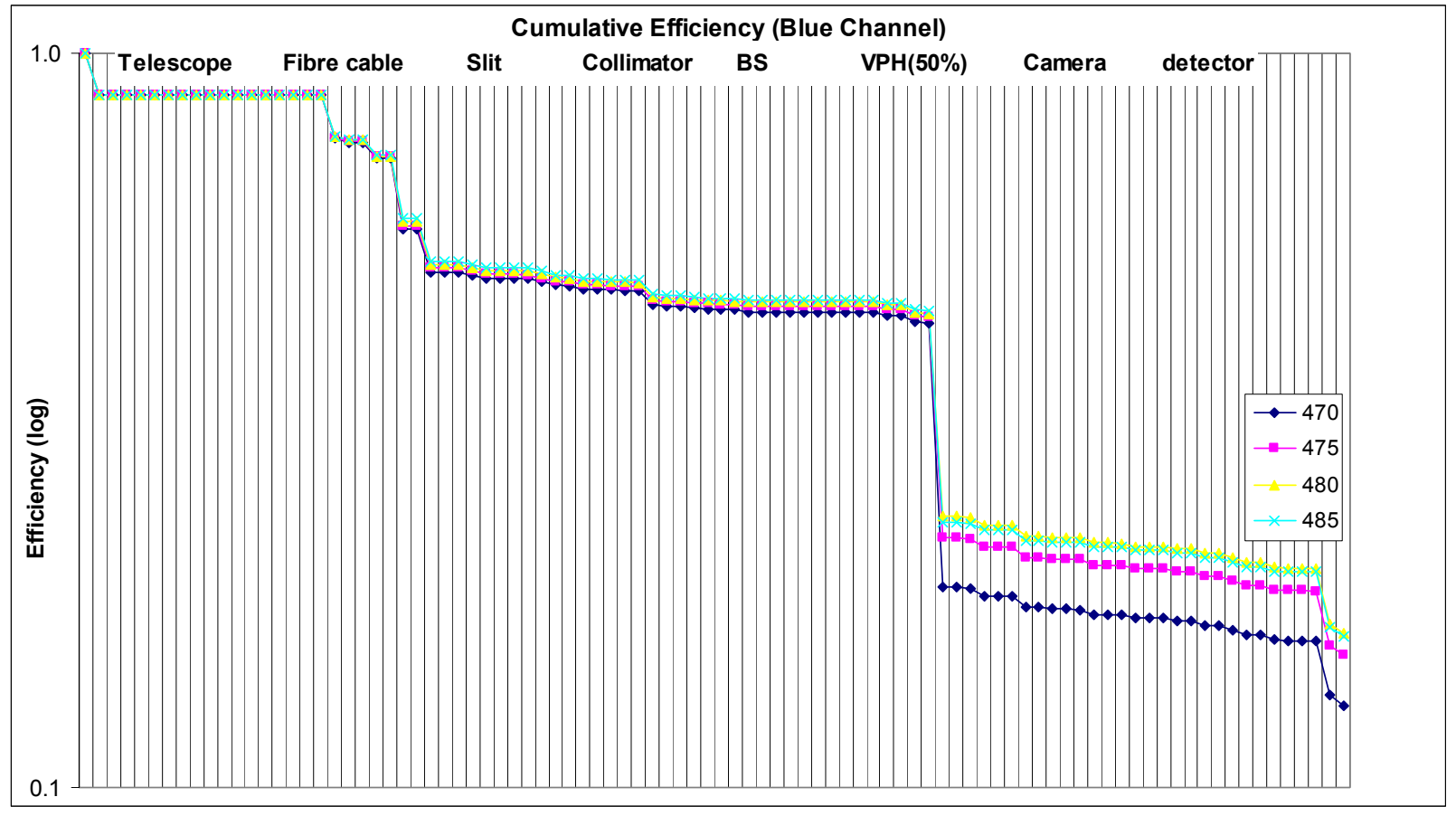

Figure 12. Cumulative efficiency (log scale) for the blue wavelength of the AAT and HERMES spectrograph at a resolution 30,000 
The resolution is predicted for both medium and high resolution of the pixilated image on the detector. This is done with the use of ZEMAX software in combination with a developed data simulation program that allows resolution prediction as a function of wavelength and the spatial and spectral position on the detector.

Scattered light is analysed for both surface scattering and for the grating properties. With the use of the software FRED masks and baffles have been designed that are placed throughout the instrument. One such baffle is placed at the 0th and reflective -1 order of the VPH grating. To minimize scattered light from hitting the detector a black honeycomb baffle has been designed. Another important aspect is the VPH grating ghost that is typical for VPH gratings used close to its Littrow configuration where the angle of incidence is equal or close to the angle of diffraction. For the AR coated blue VPH grating the ghost image has an estimated brightness of maximum $0.08 \%$ of the total light incident on the detector, depending on the actual reflective properties of the AR detector. As this ghost image is about the size of the imaged fibre, this ghost only affects a small part of the spectrum. The ghost intensity can be predicted and subtracted from the spectrum with the developed data reduction software. The performance of these aspects together is simulated with the data simulator and fed into the data reduction software.

\subsection{Data Simulator}

For the purpose of early performance verification of HERMES, it was found useful to develop an end-to-end instrument data simulator. The HERMES Data Simulator (HDS) ${ }^{11,12}$ is a command-line interface (CLI) software tool that generates synthetic detector images of each of the four cameras (spectrograph channels) for use by the engineers, instrument scientists and astronomers. The goal of the data simulator is that the simulated images are indistinguishable or closely represent that expected from the commissioned HERMES instrument. The simulation of detector images assists the development of the data reduction software to extract and process the spectra as well as test the science pipeline and verify that the science objectives can be achieved. The data simulator is sufficiently flexible to simulate other fibre-fed spectrographs by specifying the specific instrument Model Data ${ }^{11}$. The key features of the data simulator are as following:

- Ghost analysis

- $\quad$ Scattered light analysis

- $\quad$ Prediction of the medium- and high resolution modes $(\mathrm{R} \sim 30,000, \mathrm{R} \sim 50,000)$

- $\quad$ Early development of the data reduction S/W

- $\quad$ Science case validation

Science case validation: The data simulator is helpful in verifying that the instrument design and the as-built performance meets the science requirements. A preliminary analysis was conducted for the HERMES Final Design Review where the detector images were simulated for the BLUE, GREEN, RED and IR channels for a star with effective temperature of $5000 \mathrm{~K}$, surface gravity of 4.0 with solar metallicity composition. This represented the typical stellar type to be observed during the million star Galactic Archaeology survey. Exposures of 3600sec were used under 1.2 arcsec seeing conditions. The resulting efficiencies were found to be: $7.6 \%$ in the Blue, $10.4 \%$ in the Green, $10.3 \%$ in the Red, and $8.0 \%$ in the IR channels. The calculated signal-to-noise ratios (SNR) per resolution element are given in Table 1. The goal is to achieve a SNR greater than 100 for a $\mathrm{V}=14$ or brighter target. The preliminary results presented here suggest that is achievable with HERMES.

Table 1: Signal-to-no ratio (SNR) per resolution element at the centre of the detector for a star V=14

\begin{tabular}{|l|l|l|l|l|}
\hline Channel: & Blue & Green & Red & IR \\
\hline SNR & 121 & 173 & 194 & 194 \\
\hline
\end{tabular}

\section{GALACTIC ARCHEOLOGY SURVEY, GALAH}

The goal of Galactic Archaeology is to reconstruct the lost substructures of the proto-galaxy, thereby obtaining a detailed physical picture of the formation and evolution of the Milky way. The Galactic Archeology with HERMES (GALAH) survey is designed to carry out such a task for the first time using the chemical tagging technique as a means to exploit the vast chemical inventory of the disk stars. This multi-year observational program will use HERMES to obtain detailed 
elemental abundances and precise radial velocities for a million stars. Elemental abundances representing the main nucleogenesis processes in both dwarf and giant stars will allow chemical tagging of the individual stars to their original formation event, and accurate velocities will permit differentiation of stars by their dynamics.

The resulting data sets will yield a comprehensive view of the formation and evolution of the Galactic disk, enabling the reconstruction of the original stellar building blocks of the Galactic disk and address the following basic questions:

- What were the conditions of star formation during early stages of Galaxy assembly?

- When and where were the major episodes of star formation in the disk and what drove them?

- To what extent are the Galactic thin and thick disks composed of stars from merger events?

- Under what conditions and in what types of systems did the accreted stars form?

- How have the stars that formed in situ in the disk evolved dynamically since their birth?

- Where are the solar siblings that formed together with our Sun?

Additionally the GALAH data and data products provide the opportunity to address a range of science topics. Some of these include:

- Galactic star formation, structure, chemical and dynamical evolution

- $\quad$ Satellite accretion and mergers

- Stellar physics

- $\quad$ Magellanic Clouds

This Australian-led survey is planned to commence in 2013, soon after the successful commissioning of HERMES. The main survey anticipates to use $>100$ nights per year over a period of $3-5$ years. Various working groups are handling the survey preparations, from target selection to analysis to storage and data release.

Of particular relevance is the Input catalogue group, investigating the target selection and observation strategy. This includes investigating the target density over the $2 \mathrm{dF}$ two-degree field of view at various limiting magnitudes. The HERMES efficiency as well as the maximum magnitude range observable without fibre cross-talk plays a major part in deciding the survey magnitude limit as well as observing strategy, such as exposure times and field repeats.

The abundance analysis group is developing automated software to derive accurate measurements of stellar chemical abundances for the million stars targeted by GALAH. The analysis pipeline developed at ANU will interface with the reduction software developed at the AAO to ensure the required data quality is met which will enable abundance measurements to a sufficient accuracy. Using the HERMES simulator, the AAO software team and GALAH astronomers are trialing the end-to-end data handling system, including investigating the effects of the high-resolution slit-mask.

\section{CONCLUSIONS}

The AAO is well on its way to deliver the HERMES spectrograph in 2013. HERMES will provide spectra for the GALAH, Galactic Archeology survey, the first major attempt to create a detailed understanding of galaxy formation and evolution by studying the history of 1 million stars in the Milky Way. The integration is proceeding well: The collimator mirror is mounted to the completed frame and the first blue VPH grating has been developed. The blue camera is currently being assembled and the detector has been tested and verified as a grade zero device. First (test) light of the assembled blue channel is expected within the next three months. With this blue channel we will produce the spectral content of the sun for the use of a complete data reduction and chemical abundance calculation. The remaining three channels will be constructed in succession directly after the blue channel testing. The required signal to noise ratio remains challenging, especially for the blue channel. The performance specification of all components is set high and monitored closely. This is particularly the case for the fibre cable and the optical coatings.

At the AAT, the fibre cable has been assembled and its tested performance shows the efficiency has greatly improved in comparison to the existing cable. This enables shorter exposure times compared to the existing fibre cable and a higher signal to noise ratio. The scheduled fibre installation date is October of this year. The spectrograph room that accommodates and insulates the large spectrograph, has been realized. 


\section{REFERENCES}

[1] Barden, S. C., Jones, D. J., Barnes, S. I., Heijmans, J., et al., "HERMES: revisions in the design for a high resolution multi-element spectrograph for the AAT”, Proc. SPIE 7735, 773509-773509-19 (2010).

[2] Barden, S., Bland Hawthorn, J., et al., "Concepts for a high-resolution multi-object spectrograph for galactic archeology on the Anglo-Australian Telescope", Proc. SPIE 7014, 70144J-70144J-12 (2008).

[3] Brzeski, J., Gers, L., "Hermes - the engineering challenges", Proc. SPIE 8446-30 (2012)

[4] Heijmans, J.A.C., Gers, L., Faught, B., "Design and development of the high-resolution spectrograph HERMES and the unique volume phase holographic gratings", Proc. SPIE 8167A-45 (2011)

[5] Waller, L, Barton, J, Mayfield, D, Griesbach, J, “AAO2: a general purpose CCD controller for the AAT”, Proc SPIE5499-51, (2004)

[6] Case, S., Gers, L., Brezki, J., "Design and development of the fibre cable and fore optics of the HERMES spectrograph for the Anglo-Australian Telescope (AAT)", Proc. SPIE 8125, 8125-03 (2011).

[7] Farrell, T.J., Shortridge, K., Bailey, J. A., "DRAMA - An environment for Instrumentation Software”, Bulletin of the AAS, Volume 25, No 2, (1993).

[8] Shortridge, K., Farrell, T.J., Bailey, J.A., Waller, L.G., "The Data Flow System for the AAO2 Controllers", Proc. SPIE 5496, 463 (2004)

[9] Shortridge, K., Vuong, M. "Faking it for pleasure and profit: the use of hardware simulation at AAO", Proc. SPIE 7740, 774008 (2010).

[10] Cannon R., "The calibration and spectrophotometric performance of 2dF", AAO Newsletter Feb. 2000, pp.14-15

[11] Goodwin, M., Farrell, T., Smedley, S., Heald, R., Heijmans, J., De Silva, G., "The AAO Fiber Instrument Data Simulator," Proc. SPIE 8446-84, (2012) in press.

[12] Goodwin, M., Smedley, S., Barnes, S., Farrell, T., Barden, S., "Data simulator for the HERMES instrument," Proc. SPIE 7735, 77357U (2010).

[13] Sharp, R., Birchall, M. N., Optimal Extraction of Fibre Optic Spectroscopy, Publications of the Astronomical Society of Australia, Volume 27, Issue 1, pp. 91-103.

[14] Sharp, R., Hannah Parkinson, H., "Sky subtraction at the Poisson limit with fibre-optic multi-object spectroscopy", MNRAS November 2010, Volume 408, Issue 4, pages 2495-2510,

[15] Arzoumanian, Z., Holmberg, J., Norman, B., "An Astronomical pattern-matching algorithm for computer-aided identification of whale sharks", Journal of Applied Ecology 2005, 42, 991-1011.

[16] Dahl, J., et. al., "Algorithms and software for total variation image reconstruction via first-order methods", Numer Algor, 53, 67-92, 2010

[17] www.aao.gov.au/HERMES 